\author{
JAN ILUK \\ Uniwersytet Śląski w Katowicach \\ jan.iluk@us.edu.pl \\ ORCID: 0000-0003-2539-1936
}

\title{
Methodische Probleme beim Einsatz von Fragen im Fremdsprachenunterricht
}

\author{
Methodological problems of using questions \\ in foreign language teaching
}

\begin{abstract}
The author discusses the status and role of questions in foreign language teaching. Although they are essential tools of managing the learning process, the topic does not enjoy much interest in the methodological literature. Therefore it seems advisable to discuss the methodological problems of foreign language teaching in a broader context including both the activation of learners and teachers with questions and application of questions while working with a foreign language text.
\end{abstract}

KEYWORDS: Typology of questions, functions of questions, methods of asking questions in foreign language teaching.

SCHLÜSSELWORTE: Fragentypologie, Fragenfunktionen, Methodik der Fragenstellung im FSU.

\section{EINFÜHRUNG}

In der Monographie Nauka języków obcych. Od praktyki do praktyki diskutiert Waldemar Pfeiffer die Funktionen des Lehrers als Designer glottodidaktischer Prozesse (Pfeiffer 2001: 121-123). In seinen Ausführungen weist er auf die besonderen Funktionen von Kreativität, Aktivität, Steuerung und Kontrolle hin. An seine Überlegungen anschließend, möchte ich den Status und die Rolle der Fragen im Fremdsprachenunterricht eingehender behandeln, weil sie die wichtigsten Werkzeuge des Lehrers/der Lehrerin zur 
Steuerung der Lernprozesse sind. Trotzdem erfreut sich dieses Thema in der methodischen Fachliteratur keines größeren Interesses. Es fehlen nämlich zusammenfassende Darstellungen des Problems nicht nur in methodischen Lehrerhandreichungen, sondern auch in Fachlexika (vgl. Hallet \& Königs 2013; Huneke \& Steinig 1997; Heyd 1990; Bausch u.a. (2007); Jung 2009)1. Dies ist ein berechtigter Grund dafür, den methodischen Fragen der Fragestellung im Fremdsprachenunterricht in einem umfangreicheren Kontext nachzugehen.

\section{AKTIVIERUNG DER LERNENDEN MITHILFE VON FRAGEN}

Die Unterrichtsmethode, z.B. in Form eines (Mini-)Vortrags (Frontalunterricht), die oft bei der Vermittlung neuer Inhalte eingesetzt wird, setzt Wissenstransfer voraus sowie die Notwendigkeit, das vermittelte Wissen zu speichern und wiederzugeben. Ihre allgemein bekannte Schwäche besteht darin, dass sie die Aufmerksamkeit und Neugier der Lernenden weitgehend reduziert und somit eine passive Haltung hervorruft. Wenn man Lernenden ein bestimmtes Wissensquantum vermittelt, bildet man bei ihnen nicht die Fähigkeit aus, sich dieses Wissen durch Fragen zu erarbeiten. Die interaktiven und induktiven Unterrichtsformen beruhen jedoch gerade auf einem Frage-Antwort-Spiel, wobei die Fragen vom Lehrer gestellt und die Antworten von den Lernenden gegeben werden.

Die Art und Häufigkeit der Fragestellung ist mit dem Lehrstil verbunden. Verschiedene Untersuchungen zeigen, dass während einer Unterrichtsstunde 30 bis 120 Fragen gestellt werden (Seifried \& Sembill 2005: 231, Kerry 1987). ${ }^{2}$ Darüber hinaus entscheidet die Lehrkraft, wer sich wie lange und zu welchem Thema äußern darf. Unter diesen Bedingungen haben die Lehrerfragen mehrere negative Folgen:

1. Die Lernenden haben wenig Einfluss auf ihre kognitive Haltung sowie auf die Interaktionsformen im Klassenzimmer. Das führt zu einer Passivität, die selbständiges Denken behindert.

2. Die gestellten Fragen wecken normalerweise keine natürliche Neugier an dem behandelten Thema.

3. Die gestellten Fragen sind Ausdruck des Rechts des Lehrenden, die Lernenden zu kontrollieren bzw. diese zu disziplinieren. In solchen Si-

${ }^{1}$ Eine positive Ausnahme in der deutschsprachigen Fachliteratur ist das Lehrbuch von G. Storch (1999: 311-315).

2 Ein deutscher Anglist, Helmut Heuer, stellte im Rahmen seiner Hospitationen fest, dass ein Englischlehrer im Unterricht 25 bis 58 Fragen stellt (Heuer 1983). 
tuationen bewirken Fragen Stress und folglich eine Abwehrreaktion in Form von verschiedenen Vermeidungsstrategien.

4. Eine übermäßige Zahl von Fragen im Vergleich zu den erteilten Antworten bringt die Lernenden in Verlegenheit und entmutigt sie. Dies führt zu einer Reduktion der natürlichen kognitiven Neugier, die besonders dann auftritt, wenn die Fragen als banal, zwecklos oder autoritär wahrgenommen werden.

Die kritischen Anmerkungen betreffen vor allem Fragen, die nur eine Antwort implizieren. Sie kann oft aus einem Wort bestehen ${ }^{3}$. Es sind wohlgemerkt die in einer Unterrichtsstunde am häufigsten gestellten Fragen. Eine große Anzahl von Fragen zeugt eher von einem autoritären Lehrstil und einer dominierenden Rolle des Lehrers. Was besonders bedenklich ist, ist, dass ihre Zahl - wie sich oft zeigt - nicht mit deren Qualität korreliert. Es dominieren nämlich sog. display questions: geschlossene Fragen, deren Antworten dem Lehrer im Voraus bekannt sind. Sie werden ausschließlich zum Überprüfen oder zur Reproduktion des früher vermittelten Wissens gestellt. Im Fremdsprachenunterricht haben sie geradezu einen rituellen Charakter und werden von Schülern als Kontrollform des Textverstehens oder als ein Versuch der Textrekonstruktion empfunden 4 . Die Erteilung einer korrekten Antwort ist bekanntlich nicht nur eine Frage des Textverstehens, sondern sie hängt von der individuellen Fähigkeit ab, die Textinformationen zu speichern, d.h. von der Kapazität des Arbeitsgedächtnisses. Das Problem spitzt sich zu, wenn Schüler nicht nur über eine begrenzte Kapazität ihres Arbeitsgedächtnisses verfügen, sondern auch dann, wenn sie ein hohes Stressniveau während der Antwort empfinden, wodurch die Abrufprozesse wirksam gestört oder sogar blockiert werden. Deswegen nahmen Vertreter der Reformpädagogik schon vor 100 Jahren gegenüber den Lehrerfragen eine sehr kritische Stellung ein. Ihr Vertreter, Hugo Gaudig, hat auf den Widerspruch hingewiesen, der im Unterricht immer wieder zu beobachten ist. Seinen sehr kritischen Standpunkt hat er wie folgt formuliert:

Die Frageform ist eine künstliche Form der Erregung geistiger Energie; eine Schulform, die das Leben so gut wie gar nicht kennt. Im Leben wird man nicht von jemand gefragt, der uns das wissen lassen will, was er weiß, sondern wenn man uns fragt, dann will der Fragende von dem das wissen, was er nicht weiß. Sieht man also die Lernformen darauf an, ob sie auch der Schule der Fortbildung dienen, so ist die Frage so untauglich wie möglich (Gaudig 1963, nach Giel 1968: 45).

${ }^{3}$ In solchen Situationen erzwingen die Lehrperson oder die Lehrbücher eine Vollsatzantwort, was der natürlichen Kommunikation und den Prinzipien des kommunikativen Lehrens widerspricht.

${ }^{4}$ In neueren Lehrbüchern wird der Verständnisgrad des Textes auch mit anderen Techniken verifiziert. 
Aus diesem Grund sind Lehrerfragen, nach Gaudig (1963: 13), die größten Feinde der Selbständigkeit der Schüler. Trotz dieser so kritischen Beurteilung sind Fragen ein integrales Element jeder Lehraktivität. Werden sie in den von Pfeiffer genannten Funktionen eingesetzt, so sollte man immer wieder daran denken, dass ihr negativer Einfluss auf die Haltung und Motivation der Schüler möglichst reduziert werden sollte. Um dieses zu bewirken, braucht man Wissen um Frageformen und -funktionen sowie Fragestellungstechniken.

\section{URSACHEN FÜR NICHTSTELLEN VON FRAGEN IM (FREMDSPRACHEN)UNTERRICHT}

Um aus eigener Initiative Fragen zu stellen, braucht man kognitive Neugier und Wissbegier, d.h. das Bedürfnis, etwas zu verstehen, sowie den Handlungswillen, dieses Bedürfnis zu erfüllen (Pobojewska 2014: 3). Die Lehrbuchtexte, besonders auf Anfängerniveau haben den Nachteil, dass sie keine kognitive Neugier wecken. In diesem Kontext möchte ich die zutreffende Aussage von Leszek Szkutnik von 1994 über die Qualität der Lehrbuchtexte anführen: „(...) ein realistischer Text, den man in den sog. neuesten Lernbüchern finden kann, hat (...) den Nachteil, dass es sich wenig lohnt, darüber nachzudenken. ${ }^{5}$ Und wenn der Inhalt der angebotenen Texte für die Lernenden kognitiv wenig attraktiv ist, ist kaum zu erwarten, dass sie zu Fragestellungen angespornt werden. Ein wesentlicher Grund für die fehlende Angewohnheit, Fragen zu stellen und zugleich für die fehlende kognitive Neugier, besteht darin, dass Lehrbuchtexte oft keine Titel haben - die, wie man weiß, Fragen evozieren und vor der Arbeit mit dem jeweiligen Text zur Bildung von Fragen genutzt werden können ${ }^{6}$. $\mathrm{Zu}$ den weiteren Gründen für die mangelnde Fragehaltung der Lernenden kann gezählt werden:

- die unzureichende Fähigkeit, sprachlich korrekte Fragen zu formulieren ${ }^{7}$,

- die Schwierigkeit, eigene Verstehensleistungen selbst einzuschätzen",

\footnotetext{
5 Übersetzt von J.I.

6 Über den Einfluss von den durch Titel evozierten Fragen auf die Effizienz der Informationsspeicherung, das Wecken der Neugierde und die Steigerung der Motivation in Iluk (2016).

${ }^{7}$ Auf der Anfangsstufe wird die Fragenbildung erst gelernt und ihre Bildung ist mit verschiedenen Problemen, je nach der Sprache verbunden.

${ }^{8}$ Aus deutschen Untersuchen geht hervor, dass das subjektiv empfundene Verständnisniveau bezüglich der Lehrerinstruktionen mit dem tatsächlichen Zustand nicht übereinstimmt. Es ist in der Regel sehr oberflächlich und kann zur Bildung falscher Regeln führen (WißnerKurzawa 1987). Deswegen sollte man Textverständnisfragen nach den CCQ-Prinzipien formulieren.
} 
- die Schwierigkeit bei der Identifizierung der im Text enthaltenen Informationen, die man erfragen könnte,

- fehlendes Vertrauen und ungünstiges Lernklima, um im Unterricht Fragen stellen zu wollen,

- die unterstellten Wissensdefizite der Lehrer/Lehrerinnen, die eine kompetente Erteilung einer Antwort verhindern könnten,

- Angst, den Unterricht zu stören,

- die aus den niedrigeren Bildungsstufen oder anderen Fächern übernommene Angewohnheit, keine Fragen zu stellen',

- fehlendes Wissen zu einem bestimmten Thema,

- Angst vor sog. Imageverlust ${ }^{10}$.

Um Fragen zu stellen, brauchen die Lernenden nicht nur Mut, sondern auch ein Klima des Vertrauens. Indem der Lernende um Erklärung bittet, besonders bei Fremdsprachenangelegenheiten, z.B.:

What does sponge mean in Polish?

How do you spell this word?

How do we divide the word 'midday'?

signalisiert er/sie ein Sprachproblem und hegt die Hoffnung, eine Hilfe für dessen Lösung zu bekommen. Jedoch ohne Mut und aus Angst vor Imageverlust stellt der/die Lernende solche sprachbezogenen Fragen nicht. Lernende sind davon überzeugt, dass Fragen eher peinlichen Wissensdefizit oder mangelnde Vorbereitung signalisieren, was wiederum vom Lehrenden negativ beurteilt werden könnte. So eine Einstellung ist grundsätzlich falsch, weil Fragen zu empfundenen Sprachproblemen authentischen Charakter haben. Außerdem sind sie eine wichtige Kommunikationsstrategie in außerschulischen Situationen, weil sie kognitive Wachsamkeit ausdrücken und von besonderer Aufmerksamkeit zeugen. Wenn dem so ist, sollte man die Lernenden davon überzeugen und sie daran gewöhnen, dass Fremdsprachenunterricht eine natürliche Situation darstellt, in der eigene Sprachprobleme thematisiert und gemeinsam Lösungen gefunden werden, Vertrauen aufgebaut und ein positives Klima für Lernprozesse geschaffen werden. Der Lehrende sollte dabei gegenüber solch gestellten Fragen eine positive Einstellung demonstrieren und die fragende Haltung der Lerner zu schätzen wissen.

Zur Stellung von Fragen braucht man neben Mut auch Wissen. Aus eigener Erfahrung folgt, dass Lernende Fragen zu Textstellen stellen, wenn

${ }^{9}$ Unter Berufung auf internationale Untersuchungen weist Żydek-Bednarczuk (1995: 101) darauf hin, dass der Koeffizient der interrogativen Interaktion polnischer Schüler lediglich 1,25 beträgt, während er in Großbritannien bei 43 und in den Vereinigten Staaten bei 34 Prozent liegt.

${ }^{10}$ Dieses Motiv wurde in einer spontan durchgeführten Umfrage von vielen Studenten genannt. Dies bestätigt u.a. folgendes Zitat: In naturwissenschaftlichen Fächern, die nie zu meinen Stärken gehörten, vermied ich Fragen, weil ich lieber unbemerkt bleiben wollte. 
die Informationen explizit und verständlich ausgedrückt wurden. Es sind meistens Fragen, auf die man im Text die Antworten leicht finden kann. Sie stellen dagegen keine Fragen, die auf Inferieren oder Elaborieren beruhen würden. Darüber hinaus bereiten ihnen Fragen dieser Art merkliche Schwierigkeiten. Es gibt zwei Gründe dafür:

1. Lehrende stellen oft selbst keine solchen Fragen. Ihre Frequenz beträgt im Durchschnitt 4\% (Niegemann \& Stadler 2001: 173). Es handelt sich hier um sog. deep-reasoning-questions. Diese Erkenntnis erklärt die fehlende Erfahrung der Studenten in diesem Bereich.

2. Im praktischen Fremdsprachenunterricht ist die Kunst der Fragestellung kein explizites Ziel der Sprachausbildung, was mir von einer Studentengruppe bestätigt wurde, die ich als Methodiker in ihrem Magisterstudium betreut habe ${ }^{11}$.

\section{EINSTELLUNG DER LEHRENDEN ZU DEN VON LERNENDEN GESTELLTEN FRAGEN}

Fragen der Lernenden sind wünschenswert, soweit sie von kognitiver Neugier zeugen und den geplanten Unterrichtsverlauf nicht stören (sog. smart questions). Fragen der Lernenden zum Lerngegenstand bilden den Rahmen für weitere Aktivitäten während des Unterrichts, wecken Interesse am angesprochenen Thema und beeinflussen die Lehr- und Lerneffizienz positiv. Hospitationen bestätigen jedoch, dass Schüler und sogar Studierende während des traditionell geführten Unterrichts aus eigener Initiative so gut wie keine Fragen stellen.

Es kann besonders in Unterrichtsstunden mit einem präzisen Zeit- und Inhaltsplan passieren, dass Schülerfragen von der Lehrkraft als beabsichtigte Störung empfunden werden. In solchen Fällen wird angenommen, dass sie den Lehrenden vom geplanten Unterrichtsablauf abbringen oder sogar den Unterrichtsablauf absichtlich stören und den Lehrer irritieren sollen, was als ein offensichtlicher Konfrontationsversuch gedeutet werden kann. Fragen ${ }^{12}$, wie etwa: ${ }^{13}$

${ }^{11}$ Diese Information wurde von Studierenden des 1. Jahres eines pädagogischen Magisterstudiums am Institut für Germanische Philologie der Schlesischen Universität Katowice erteilt (2018). Unter den Studierenden wurde eine Umfrage durchgeführt, in der sie u.a. berichteten, in welchen Unterrichtssituationen sie Fragen gestellt haben oder was sie davon abhielt.

${ }^{12}$ Die Beispiele stammen aus dem Blog Angielska Herbata. Wpis: Jakich pytań nie zadawać nauczycielowi jezyka angielskiego? (Englischer Tee), Eintrag: Welche Fragen sollte man einem Englischlehrer nicht stellen? http:/ /angielskaherbata.pl/2016/03/12/10-pytan/ (Zugang am 12.05.2018).

${ }^{13}$ Die Frage Wozu? ist eine Frage nach dem Sinn, Ziel oder praktischen Nutzen einer Lernaktivität. Sie sollte als Signal des Lernenden interpretiert werden, dass er / sie ein vorübergehendes Problem mit Motivation oder Lernlust hat. 
- Auf Englisch?

- Im ganzen Satz?

- Soll ich die Aufgabe vorlesen?

- Soll ich von vorne lesen?

- Bekommen wir dafür eine Note?

- Müssen wir das machen?

- Gebrauchen das die Engländer wirklich?

- Wozu brauche ich das?

- Wofür ist diese Hausaufgabe?

- Wird das im Test drankommen?

- Jetzt?

bringen die Lehrer oft aus der Fassung oder führen zu starken Irritationen, die nicht selten in ironischen oder sarkastischen Kommentaren zum Ausdruck gebracht werden: 14

- Reaktion auf die Frage: "Auf Englisch?" - Was soll das wieder! Wir haben heute doch eine Ballettstunde und werden Kniebeugen an der Decke üben. Zwischen Kronleuchter und Gardinenstange kannst du dann ein altfranzösisches Chanson deklamieren.

- Reaktion auf die Frage: „Im ganzen Satz?" - Nein, in Einzelworten. Wir lernen doch nicht dafür, dass du deinen Kopf mit ganzen Sätzen kaputt machst! Full sentences are sooo 2015!

- Reaktion auf die Frage: "Und warum diese Hausaufgabe?" - Damit du die Gelegenheit hast, das zu üben, was ich dir vor einer Minute beinahe mit Gewalt in den Kopf gesteckt habe.

Die Gründe, warum solche organisatorischen Fragen gestellt werden, können verschieden sein. Manche Lehrer interpretieren sie als einen Versuch, den Stundenverlauf gezielt zu stören oder Zeit für sich zu gewinnen. Ist der Grund ein anderer, so sollte der Lehrende darüber nachdenken und sich unbedingt einer sarkastischen Reaktion enthalten. Studenten einer pädagogischen Studienrichtung haben die Motive für die Stellung der oben zitierten Fragen wie folgt erklärt:

- Ignoranz,

- zeitweilige Hörstörung,

- Langweile oder Müdigkeit in der Stunde15,

- andere Denkrichtung ${ }^{16}$,

${ }^{14}$ Die Auswahl der zitierten Reaktionen irritierter Lehrer stammt aus dem Blog Angielska Herbata (Englischer Tee), Eintrag: Welche Fragen sollte man einem Englischlehrer nicht stellen? (Zugang am 12.05.2018).

${ }^{15}$ Lernende finden es langweilig, wenn man sie z.B. reproduktive Übungen ins Heft abschreiben lässt.

${ }^{16}$ Es zeigt sich, dass Lehreranweisungen für viele Schüler nicht präzise genug oder sogar unklar sind. In solchen Situationen - wie eine Studentin berichtete - diskutieren die Schüler 
- langsamere Denkgeschwindigkeit oder Konzentrationsverlust ${ }^{17}$,

- kein Verständnis für die vorgeschlagene Lernaktivität 18 ,

- kein Interesse an der vorgeschlagenen Lernaktivität ${ }^{19}$,

- Sorge um eigene Noten ${ }^{20}$,

- Versuch, die Gruppe zum Lachen zu bringen, um Stress abzubauen,

- Versuch, die Lehrperson auf die Überforderung oder Ermüdung der Schüler hinzuweisen ${ }^{21}$.

Aus den genannten Motiven geht hervor, dass die Gründe für das Stellen der weiter oben erwähnten Schülerfragen grundverschieden sein können. Manche davon sind so seriös, dass der Lehrende sie weder ignorieren noch ihnen negative Intentionen unterstellen sollte. Es zeigt sich, dass Schüler von ihren Lehrern und Lehrerinnen immer gut überlegte und keine spöttischen Antworten erwarten.

\section{DIDAKTISCHE FUNKTIONEN VON LEHRERFRAGEN}

In einem Seminargespräch mit Lehramtsstudenten zum Thema Lehrerfrage gaben sie zu, dass sie nur zwei Formen von Aussagen mit erotetischem Charakter unterscheiden:

- Entscheidungsfragen,

- detaillierte Fragen, die in Grammatikbüchern auch Ergänzungsfragen genannt werden.

untereinander: „Was sollen wir eigentlich machen?". Eine andere Studentin schrieb: Ich habe nur dann Fragen an den Lehrer gestellt, wenn die Aufgabe für mich unklar war.

17 Ca. ein Drittel der Lehrer erklärt den Lernstoff zu schnell, besonders für Schüler, deren Reaktionen langsamer sind. Darüber hinaus wirkt sich der empfundene Zeitdruck negativ auf die Lerneffizienz aus. In solchen Situationen sind spöttische Kommentare besonders unangebracht.

${ }^{18}$ Meine eigenen Erfahrungen wurden von den Studenten bestätigt, nämlich dass Lehrer nie den Sinn der Aufgaben erklärt haben, die die Schüler machen sollten.

${ }^{19}$ Das mangelnde Interesse an Lernhandlung motiviert dazu, Vermeidungsstrategien intensiver einzusetzen.

${ }^{20}$ Das Motiv für die Frage: Wird das benotet? oder Kommt es in dem Test vor? ist - wie es sich herausstellt - die besondere Sorge um eine gute Note. Um diese zu erreichen, ist nicht selten eine größere Anstrengung nötig. Die Perspektive, eine gute Note zu bekommen, verstärkt die Motivation, die Aufgabe fleißig auszuführen. Diese Einstellung zeugt von einer dominierenden äußeren Motivation und ist stark instrumental gekennzeichnet (vgl. Iluk 2013: 68). In dem Falle, dass die Motivation zum Lernen ganz fehlt, implizieren diese Fragen eine minimalistische Einstellung zum Lernen. Wie eine der Studentinnen erklärte, die diese Strategie oft eingesetzt hatte, ging es ihr darum, herauszufinden, welchen Stoff man unbedingt beherrschen muss, um wenigstens die niedrigste positive Note zu bekommen. (d.h., die Note ausreichend).

${ }^{21}$ Dies wird normalerweise mit einer Stimme ausgedrückt, die ein Mitleidsgefühl beim Lehrer hervorrufen soll. 
Informationen, die Studenten in ihren Lehrveranstaltungen zur beschreibenden Grammatik vermittelt bekommen, sind jedoch nicht ausreichend, um die Kunst der Fragestellung im FSU zu erwerben. Hierfür braucht man also ein viel breiteres Wissen. Im Folgenden wollen wir insbesondere auf die methodischen Aspekte der Fragestellung eingehen.

\section{FUNKTIONEN VON LEHRERFRAGEN}

Die Häufigkeit der Lehrerfragen ergibt sich aus deren Funktionenvielfalt. Ihre besondere Rolle beruht darauf, dass sie 22 :

- die kognitive Aufmerksamkeit der Lerner besonders beim Frontalunterricht stimulieren und fokussieren,

- zum Nachdenken und zur Reflexion inspirieren,

- Interaktion im Unterricht initiieren und Kommunikation unterstützen,

- Schüler in den Unterrichtsverlauf miteinbeziehen,

- beim Verstehen gehörter oder gelesener Informationen helfen,

- den Wissensbestand und das Verständnisniveau gehörter oder gelesener Informationen verifizieren,

- die Beherrschung von bestimmten Fähigkeiten und Fertigkeiten überprüfen lassen,

- die Erinnerung an Fakten oder sprachliche Mittel zum Ausdruck bestimmter Inhalte erleichtern,

- es erleichtern, Informationen zu speichern,

- helfen, den Lehrprozess zu organisieren.

Die erwähnten Funktionen legen nahe, wie wichtig die Lehrerfragen im Unterricht sind. Anhand von Fragen steuert die Lehrperson den Lernprozess, beeinflusst dessen Tempo, die Schüleraktivitäten und die Art der implizierten Antworten. Aus diesem Grund sollte der Fremdsprachenlehrer nicht nur die Funktionen der Fragen gut kennen, sondern sich auch ihrer Rolle in einer konkreten Lehrsituation bewusst sein. Er sollte auch wissen, welche Frageform für die jeweilige Lernsituation und im Zusammenhang mit dem angestrebten Ziel optimal geeignet ist.

\section{FRAGETYPEN}

In der Fachliteratur unterscheidet man zwischen geschlossenen und offenen Fragen. Storch (1999: 312) weist darauf hin, dass Fragen sowohl auf der syntaktischen als auch auf der semantischen Ebene geschlossen oder

${ }^{22}$ Vgl. 99 Stichwörter für den Fremdsprachenunterricht. Fragen. (online, Zugang 18.05.2018). 
offen sein können. Syntaktisch geschlossene Fragen implizieren normalerweise eine kurze und syntaktisch vorhersehbare Antwort, besonders dann, wenn der Lernende im vollen Satz antworten soll. Die Antwort auf solche Fragen ist in der Regel in deren Inhalt vorgegeben. z.B..:

Gehst du nach Hause? Ja (, ich gehe nach Hause). ${ }^{23}$

Welche Antwort ist richtig: a oder b? (Richtig ist die Antwort) $b$.

Semantisch geschlossene Fragen, auch Konvergenzfragen oder Protokollfragen genannt, betreffen allgemein bekannte Sachverhalte und deswegen ist die Antwort darauf vorhersehbar. Man setzt sie häufig in der Anfängerstufe ein, weil sie ein effizientes Mittel der Schüleraktivierung sind. Man setzt sie auch oft in Übungen ein, die auf das Abrufen von Informationen aus dem Gedächtnis abzielen, oder bei jeder Art von Wissenskontrolle. Den Status von semantisch geschlossenen Fragen haben hauptsächlich Fragen nach konkreten Informationen aus einem bereits behandelten Text, vermittelten grammatischen Problemen oder lexikalischen Entsprechungen. Die dabei gebrauchten Frageoperatoren: wer, was, wen, worüber, warum usw., entscheiden über den Inhalt der Antwort, z.B.

An welcher Stelle steht das Prädikat in einem Aussagesatz?

Wie heißt Tafel auf Englisch?

Wer spricht in diesem Dialog?

Worüber sprechen sie?

Wen möchte Helga zu ihrem Namenstag einladen?

Eine Einteilung der Entscheidungsfragen hängt vom Umfang der möglichen Antworten ab. Deswegen unterscheidet man alternative und multialternative Fragen.

Syntaktisch offene Fragen determinieren dagegen keine grammatische Form der Antwort. Der Antwortende muss selbst entscheiden, wie er in grammatischer Hinsicht seine Antwort formuliert. Diese Art von Fragen verlangen allerdings eine größere Selbständigkeit und ein höheres Kompetenzniveau, z.B.:

Wie ist es zu diesem Unfall gekommen?

Wie bist du auf die Idee gekommen?

Wie findest du seine Haltung?

Was meinst $d u$, wenn $d u$ "gerecht" sagst?

Auf semantisch offene Fragen (auch Divergenzfragen genannt) gibt es verschiedene Antwortmöglichkeiten. Sie sind gleichzeitig syntaktisch offen. Die Erteilung einer Antwort darauf erfordert Wissen, Vorstellungskraft und/oder Kreativität, z.B.:

${ }^{23}$ Je nach Sprache werden die Antworten auf Fragen nach Bestätigung oder Entscheidung spezifisch formuliert: vgl. im Englischen: Do you go home? Yes, I do., im Deutschen: Gehst du nach Hause? Ja. 
Wie könnte man das angesprochene Problem anders lösen?

Wie könnte diese Geschichte anders zu Ende gehen?

Was würdest du an ihrer Stelle tun?

Eine wichtige Rolle spielen sog. Sondierungsfragen. Man kann mit ihrer Hilfe verschiedene Aspekte eines Themas erfragen, die für die jeweilige Gesprächssituation von Bedeutung sein können. Sie beschränken sich in der Regel nicht auf eine Frageform, sondern erfordern den Einsatz einer Reihe gemischter, d.h. offener und geschlossener Fragen, z.B.:

N: Was möchtest du werden?

U: Fußballspieler

N: Warum?

U: Weil ich gut Fußball spiele.

N: Gibt es noch andere Gründe, warum du Fußballspieler werden möchtest?

Im Unterricht stellt man auch eine Vielzahl von Fragen zu Organisation und Verlauf der Unterrichtsstunde ${ }^{24}$, z.B.:

Versteht ihr die Aufgabe?

Haben alle eine Textkopie bekommen?

Warum hast du dich verspätet?

Wer braucht meine Hilfe?

Aus pragmatischer Sicht haben viele organisatorische Fragen keinen interrogativen Charakter, sondern sie fungieren eher als eine vorsichtige Bitte oder eine freundliche Anweisung, z.B.:

Kannst du den Beamer anschließen?

Können wir anfangen?

Beurteilungsfragen sollen Aussagen initiieren, mit denen Lernende ihre Meinung zu einem bestimmten Thema äußern sollen. z.B.:

Was hältst du von der Aussage dieses Posters?

Wie findest $d u$ das Poster?

Hat er sich richtig benommen?

Wie beurteilt ihr die Pläne ihrer Kollegin?

\section{DIE ROLLE VON INFERENZFRAGEN UND DEREN EINFLUSS AUF DIE ARBEITSEFFIZIENZ MIT EINEM FREMDSPRACHLICHEN LEHRTEXT}

Eine durchaus motivierende und effektive Arbeitsmethode mit einem Lehrtext ist dessen Behandlung mit sog. Inferenzfragen. Ihre Rolle besteht

${ }^{24}$ Eine große Auswahl solcher Fragen für den Fremdsprachenunterricht enthalten einschlägige Wörterbücher der Unterrichtssprache (vgl. z.B. Iluk 1993; 1995). 
darin, dass dabei das Vorwissen der Lerner in die Interpretation von implizit ausgedrückten Informationen im Text miteinbezogen wird, wozu Inferenzen und Elaborationen notwendig sind (vgl. Hartl \& Piontek 1989: 11; Iluk 1998: 14-15). Die Erteilung einer Antwort auf eine Inferenzfrage verlangt eine tiefere Verarbeitung des Textes und den Einsatz komplexerer kognitiver Operationen, wie etwa Schlussfolgerungen und Begründung ausgedrückter Meinungen oder Hypothesen. Dadurch werden der Textinhalt sowie die sprachlichen Mittel besser und länger gespeichert.

Im Rahmen eines Seminars zur „Methodik des Unterrichts Deutsch als Fremdsprache" haben die Studenten von mir einen Text aus einem Lehrbuch bekommen und sollten dazu Inferenzfragen bilden. Bei der Textauswahl waren folgende Kriterien ausschlaggebend:

- niedriges lexikalisches Niveau des Textes,

- niedriges Inhaltsniveau des Textes,

- beschränkte Textlänge.

\section{Auf dem Land}

Gerd Becker wohnt mit seinen Eltern, seinen Großeltern und seiner kleinen Schwester auf einem Bauernhof auf dem Land. Jeden Morgen, Montag bis Freitag, steht er um fünf vor sieben auf. Er duscht sich, frühstückt und ist schon um halb acht auf dem Weg zur Arbeit.

Möchtest du wie Gerd auf einem Bavernhof wohnen?

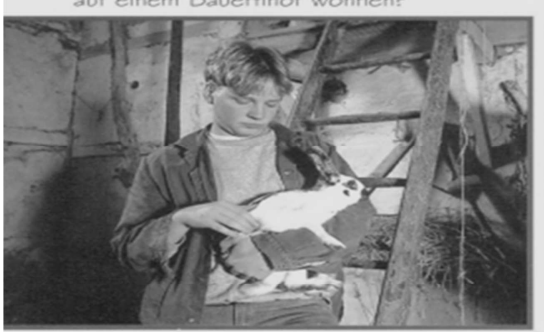

Gerd ist Azubi

bei einer Firma in Soest. Er will später

Elektrotechniker werden. Es wird noch drei

Jahre dauern, bis er mit seiner Lehre fertig ist.

Nach der Arbeit und auch am Wochenende hilft er meistens seinem Vater auf dem Bauernhof. Da gibt's immer viel zu tun. Beckers haben viele Schweine, ein paar Kühe und auch ein paar Kaninchen. Er muss oft beim Füttern helfen.

Am Abend kommt die ganze Familie zum Abendessen zusammen.

Nach dem Abendessen trifft sich Gerd oft mit seinen Freunden in einem Sportklub.

Billard ist dort das beliebteste Spiel, deshalb müssen Gerd und seine Freunde fast immer warten, bis sie spielen können. Ein anderes beliebtes Spiel ist Tischfußball.

Aber den echten Fußball spielt Gerd am Sonntag. Er und seine Freunde gehören zu einer Jugendmannschaft in Soest. Sie spielen immer gegen andere Mannschaften in der Gegend. Gerd ist der beste Spieler und schießt viele Tore für seine Mannschaft.

Manchmal kommt auch seine Freundin, sieht dem Spiel zu und gratuliert ihm, besonders wenn seine Mannschaft gewonnen hat.

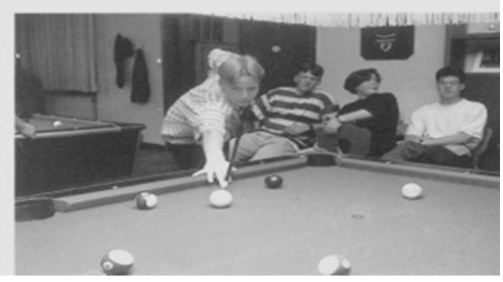

Abbildung 1. Text aus dem Lehrbuch für Deutsch als Fremdsprache (vgl. Kraft, Rybarczyk \& Schmidt 2005: 114) 
Die zwei ersten Kriterien sollten Texterschließungsschwierigkeiten auf lexikalischer und inhaltlicher Ebene ausschließen. Es ging darum, mögliche Schwierigkeiten zu eliminieren, die sich negativ auf das Ausformulieren von Fragen zum Text auswirken könnten. Es ging auch darum, zu ermitteln, wie schnell Fragen gebildet werden und worauf sie abzielen. Grundlage für dieses Experiment bildete umseitiger Text aus einem Lehrbuch für Deutsch als Fremdsprache für 15-16-jährige Lernende im polnischen Gymnasium.

Vor der eigentlichen Aufgabe wurden die Studenten gebeten, Fragen zu bestimmten Sätzen oder Satzteilen (bei zusammengesetzten Sätzen) zu bilden. Die schriftlich formulierten Fragen hatten die Form von sog. Protokollfragen $^{25}$, z.B.:

Mit wem wohnt Gerd Becker auf dem Bauernhof?

Man gebraucht sie häufig im Fremdsprachenunterricht unabhängig von der sprachlichen Stufe. Gemäß den Erwartungen bereitete diese Aufgabe den Studenten keine Schwierigkeiten. Nur in Einzelfällen konnten sie detailliertere Informationen nicht identifizieren, über die man eine separate Frage bilden konnte, z.B.:

Um wie viel Uhr steht Gerd werktags auf?

Welche Spielarten bietet der Sportklub an?

Im nächsten Schritt wurde ihnen erklärt, worauf Inferenzfragen beruhen und sie bekamen konkrete Beispiele zum gleichen Text.

Wie weit ist Gerds Wohnort von Soest entfernt?

Wie lange dauert die Ausbildung zum Elektrotechniker?

Warum will Gerd kein Landwirt werden?

Warum gibt es in Gerds Dorf keine Fußballmannschaft?

Anschließend begannen die Studenten in Paaren eigene Inferenzfragen zu bilden. Man konnte beobachten, dass die gestellte Aufgabe ihr Interesse weckte. Nach einiger Zeit machten sie jedoch die Erfahrung, dass sie ganz wenige Inferenzfragen gebildet hatten. Ihre Bildung nahm mehr Zeit in Anspruch, weil man zuerst eine tiefere Verarbeitung des Textinhalts vornehmen musste, was an der Intensität gegenseitiger Konsultationen sichtlich war. Ein wesentlicher Hemmfaktor war der von den Studenten bestätigte Erfahrungsmangel mit der Ausführung dieser Art von Aufgaben. Dies war für mich überraschend, weil die Formulierung von Inferenzfragen keine neue Spracherfahrung für Studenten auf dieser Bildungsstufe darstellen dürfte.

An einer anderen Stelle habe ich die Ergebnisse des gezielten Einsatzes von Inferenzfragen bei der Erschließung eines fremdsprachlichen Textes

${ }^{25}$ In der englischsprachigen Literatur heißen sie wh-questions, in der deutschsprachigen W-Fragen. 
(Iluk 2001) präsentiert. Sie belegten, dass die Antworten, zu denen dieser Fragetyp die Studenten anregte, spontaner, inhaltsreicher und verbal besser begründet waren. Der auf einen Lehrbuchtext bezogene Dialog mit den Lernern hatte einen merklich authentischen Charakter. Die darauf angefertigten Zusammenfassungen des Textes zeigten folgende Vorteile:

1) Die Texte waren deutlich länger im Vergleich mit den Zusammenfassungen derjenigen Studenten, die den Text mit traditionellen $\mathrm{W}$ Fragen (wer, wen, mit wem, wo, wann) bearbeitet hatten, und enthielten viele zusätzliche Informationen über die Protagonisten und ihre gegenseitigen Beziehungen.

2) Die Zusammenfassungen des Textes enthielten zahlreiche Kommentare und emotionelle Wertungen.

3) Die abgelieferten Texte hatten eine klare Erzählperspektive. Die Tempuswahl orientierte sich nicht an dem Ausgangstext, sondern an der gewählten Erzählperspektive oder den temporalen Zusammenhängen.

4) Die Texte zeichneten sich durch eine hohe Kohärenz aus.

5) Die Grundlage für die angefertigten Zusammenfassungen bildeten die gezogenen Inferenzen und Elaborationen und nicht die Struktur und der Inhalt des Ausgangstextes.

6) Die verfassten Texte kennzeichnen sich durch große Selbständigkeit und Kreativität, d.h. es wurden kaum Formulierungen aus dem Ausgangstext reproduziert.

In der Vergleichsgruppe, in der der Text traditionell behandelt wurde, konnte man in den angefertigten Zusammenfassungen keine Anzeichen von Kreativität feststellen. Sie enthielten wortwörtliche Formulierungen aus dem Ausgangstext und die Abfolge der beschriebenen Szenen war mit ihm identisch. Die Strategie beim Abfassen der Textzusammenfassung beschränkte sich de facto auf eine mechanische Informationsreduktion. Die Lösung dieser Aufgabe blieb daher unbefriedigend, die Präsentation der Zusammenfassungen vor der gesamten Gruppe weckte bei den Studenten kein Interesse und der Text selbst wurde von ihnen als langweilig beurteilt. Die Experimentalgruppe stellte dagegen fest, dass die gewählte Form der Textbehandlung inspirierend war und zur Stellungnahme motivierte. Darüber hinaus fanden sie denselben Text außergewöhnlich interessant und die geschriebenen Zusammenfassungen waren auf einem zufriedenstellenden Niveau.

\section{SCHLUSSFOLGERUNGEN}

Wenn gut formulierte Fragen die intellektuelle und sprachliche Entwicklung von Lernenden und Studierenden maßgeblich fördern und schlecht gestellt unerwünschte Ergebnisse herbeiführen, dann sind die Kenntnis ihrer 
Formen und Funktionen sowie die Beherrschung der Kunst ihrer effektiven Formulierung äußerst wichtig. Daraus sowie aus der Fachliteratur ergeben sich folgende Schlussfolgern für den FSU:

1. Man sollte Lernende dazu ermuntern, nach den Inhalten zu fragen, die für sie unverständlich sind.

2. Man sollte sich mit Fragen an die gesamte Klasse und nicht an konkrete Schüler wenden.

3. Bevor man eine Frage stellt, sollten die Schüler konzentriert sein.

4. Nachdem man eine Frage gestellt hat, sollte man, je nach Bedarf, ein paar Sekunden abwarten, damit alle Schüler, unabhängig von ihrer individuellen Reaktionszeit, Zeit für das Verstehen der Frage, die Vorbereitung der Antwort oder ihre Abstimmung mit anderen Lernenden haben. ${ }^{26}$

5. Man sollte alle Schüler bei der Antwort auf die gestellte Frage miteinbeziehen, d.h., es ermöglichen, dass eine Frage auch von Schülern beantwortet wird, die sich nicht gemeldet haben. Vielleicht kennen sie die Antwort, zeigen aber aufgrund erlebter Misserfolge keine Lust, diese zu geben.

6. Fragen sollten sich durch verschiedene Schwierigkeitsstufen sowie durch Offenheit auszeichnen. Damit verschafft man auch schwächeren Schülern die Möglichkeit, sie zu beantworten.

7. Die Antworten auf Fragen zu einem Text sollten sich nicht auf die Reproduktion der in ihm enthaltenen Sätze beschränken. Sie sollen vielmehr das Ergebnis einer kognitiven Umwandlung des Inhalts oder seiner Struktur gemäß der Möglichkeiten der Lernenden darstellen ${ }^{27}$.

8. Antworten können auch in der Muttersprache erteilt werden, falls deren Formulierung in der Fremdsprache zu schwierig wäre.

9. Man sollte keine Vollsatzantwort verlangen, wenn gemäß dem Sprachusus eine Kurzantwort möglich ist.28

10. Falls Lernende nicht imstande sind, eine richtige Antwort zu formulieren, sollten sie um Hilfe bei deren Formulierung bitten dürfen.

11. Richtige Antworten sollen mit einer akzeptierenden Geste oder verbaler Zustimmung angenommen werden.

\footnotetext{
${ }^{26}$ Aus eigenen Beobachtungen des Studentenverhaltens in Seminardebatten folgt, dass die Möglichkeit, die eigene Antwort zunächst mit einer anderen Person abzustimmen, die Sprechangst weitgehend reduziert, alle Studenten zur kognitiven Aktivität mobilisiert und eine adäquatere und sprachlich korrekte Antwort garantiert.

${ }^{27}$ Dazu kann man z.B. die Präsentationsperspektive oder die Textgattung ändern (z.B. einen Informationstext in einen Dialog umwandeln und umgekehrt) usw.

${ }^{28}$ Z.B. auf die Frage: Wer hatte Geburtstag? lautet die ausreichende Antwort Kasia., und nicht: Kasia hatte Geburtstag., oder Geburtstag hatte Kasia. Analog kann man die Frage: Warum ist Kasia abwesend? , gemäß dem natürlichen Sprachgebrauch beantworten mit: Weil sie krank ist. und nicht mit: Kasia ist abwesend, weil sie krank ist.
} 
12. Auch teilweise korrekte Antworten sind zu akzeptieren.

13. Das akzeptierende Feedback sollte man mit einer angemessenen Mäßigung dosieren.

14. Negative Reaktionen auf unkorrekte Antworten sind zu vermeiden.

15. Nach einer falschen Antwort sollte man die Frage mit den nötigen Hinweisen wiederholen.

16. Bei Bedarf sollte man Fragen so wiederholen, dass die Antwort des Schülers in eine Frage umgewandelt wird.

17. Indem man Fragepronomen oder Fragepartikel anwendet, sollte man die Schüler mit ihren spezifischen Funktionen bekannt machen.

18. Aus empirischer Untersuchung ergibt sich, dass Fragen zu Texten mit einer transparenten Struktur korrekter beantwortet werden als Fragen zu Texten, deren Struktur weniger transparent ist (Hartl. Piontek 1989: 11). Dies ist ein wichtiger Hinweis für die Wahl der Lehrtexte für den FSU.

Bei der Beantwortung von Lehrerfragen zu einem Text sollte der Schüler, je nach Niveau in der Fremdsprache, beweisen können, dass er:

- die wichtigsten Informationen verstanden hat,

- die in dem Text enthaltenen Sachverhalte nach vorgegebenen Kriterien ordnen kann,

- bestimmte Sprachhandlungen realisieren kann (z.B. eine Beschreibung durchführen, einer Auffassung zustimmen bzw. widersprechen),

- längere Textteile zusammenfassen kann,

- Tatbestände oder Ereignisse vergleichen kann,

- die in dem Text beschriebenen logischen Zusammenhänge versteht und diese darstellen kann,

- Beispiele nennen und aus bestimmten Perspektiven interpretieren kann,

- Schlussfolgerungen ziehen oder formulieren kann.

Dementsprechend dienen Fragen nicht ausschließlich der Wissenskontrolle. Sie bilden ein wesentliches Mittel zur Steuerung der mentalen Arbeit und der Unterrichtsinteraktion. Aus diesem Grunde ist das Wissen um die Formen und Funktionen von Lehrerfragen sowie die Kunst effizienter Fragenstellung ein wesentliches Element der methodischen Kompetenz von Lehrenden.

\section{LITERATURVERZEICHNIS}

Bausch, K. R. / Christ, H. / Krumm, H. J. (2007) (Hrsg.). Handbuch Fremdsprachenunterricht. (Vol. 5). Tübingen: Francke Verlag.

Gaudig, H. (1963). Didaktische Präludien. In: L. Müller (Hrsg.), Die Schule der Selbsttätigkeit. Bad Heilbrunn: Klinkhardt. 
Giel, K. (1968). Über die Frage mit besonderer Berücksichtigung der Lehrerfrage im Unterricht. In: G. Bräuer / K. Giel / W. Loch / J. Muth (Hrsg.), Studien zur Anthropologie des Lernens. Neue pädagogische Bemühungen. Bd. 36 (S. 35-64). Essen: Neue Deutsche Schule Verlagsgesellschaft. http:// www.klaus-giel.de/doc/FrageA.pdf (Zugang am 12.05.2018).

Hallet, W. / Königs, F. (2013). Handbuch Fremdsprachendidaktik. Seelze Velber: Klett/Kallmeyer.

Hartl, B. / Piontek, F. (1989). Zu Funktionen und Aufgaben von „Fragen zum Text” aus lernpsychologischer Sicht. DaF, 1, 8-114.

Heuer, H. (1983). Die Fragestellung im Fremdsprachenunterricht. In: G. Solmecke (Hrsg.), Motivation und Motivieren im Fremdsprachenunterricht (S. 228-239). Paderborn: Schöningh.

Heyd, G. (1990). Deutsch lehren. Grundwissen für den Unterricht in Deutsch als Fremdsprache. Frankfurt/M: Peter Lang.

Huneke, H. W. / Steinig W. (1997). Deutsch als Fremdsprache. Berlin: Erich Schmidt.

Iluk, J. (1993). Polsko-niemiecki stownik wyrażeń i zwrotów lekcyjnych. Warszawa: WSiP.

Iluk, J. (1998). Entwicklung der Sprachfertigkeiten aus der Sicht der neuesten Fremdsprachencurricula. Katowice: Wydawnictwo Uniwersytetu Śląskiego.

Iluk, J. (2001). Der Einkauf. Oder: Der Tag ist schon wieder hin! Integrative Fertigkeitsentwicklung mit Lehrwerktexten. Fremdsprache Deutsch. Kombinierte Fertigkeiten, 4, 19-20.

Iluk, J. (2013). Jak (de)motywujemy uczniów na lekcjach języka obcego. Jezzyki Obce w Szkole, 4, 67-74.

Iluk, J. (2016). Wpływ tytułu tekstu oraz konstruktywistycznego podejścia na efektywność przyswajania wiedzy z podręczników do nauczania geografii. Acta Geografica Silesiana, 24, 61-70.

Iluk, J. / Gabryś, D. (1995). Polsko-angielski stownik wyrażeń i zwrotów lekcyjnych. Warszawa: WSiP.

Jung, U. O. H. (2009). Praktische Handreichung für Fremdsprachenlehrer. 5. Auflage. Frankfurt/M.: Peter Lang.

Kerry, T. (1987). Classroom questions in England. Questioning exchange, 1, 32-33 .

Kraft, W. / Rybarczyk, R. / Schmidt, M. (2005). Deutsch aktuell 3. Kompakt. Poznań: Klett.

Niegemann, H. / Stadler, S. (2001). Hat noch jemand eine Frage? Unterrichtswissenschaft, 29 (2), 171-192.

Pfeiffer, W. (2001). Nauka języków obcych. Od praktyki do praktyki. Poznań: Wagros.

Pobojewska, A. (2014). Waga pytań w procesie edukacji. In: P. Mroczkiewicz / W. Kamińska (Hrsg.), Jak uczyć, by nauczyć filozofii? Refleksje akademików i praktyków (S. 107-120). Warszawa: Wydawnictwo UKSW. http://kronika.uni.lodz.pl/pliki/waga-pyta-w-procesieedukacji1-tekst-zainspirowany-cwiczeniem-berri-hessena-oraz-roberta-pilata.pdf (Zugang am15.05.2018).

Seifried, J. / Sembill, D. (2005). Schülerfragen - ein brachliegendes didaktisches Feld. Zeitschrift für Berufs- und Wirtschaftspädagogik, 101 (2), 229-245. Online: Konstanzer Online-Publikation-System (KOPS) (Zugang am 15.06.2018).

Storch, G. (1999). Deutsch als Fremdsprache - Eine Didaktik. München: Wilhelm Fink.

Wißner-Kurzawa, E. (1987). Zur Optimierung von grammatikalischen Instruktionstexten. Frankfurt/M: Peter Lang.

Żydek-Bednarczuk, U. (1995). Komunikacja dydaktyczna na lekcjach języka polskiego. Z Teorii i Praktyki Dydaktycznej Jezyka Polskiego, 14, 93-103.

Received: 21.08.2018; revised: 12.09 .2018 
